\title{
The optical mouse as an inexpensive measurement device
}

\section{T. W. $\mathrm{Ng}$}

T. W. Ng, "The optical mouse as an inexpensive measurement device," Proc. SPIE 9663, Eighth International Topical Meeting on Education and Training in Optics and Photonics, 96631J (6 October 2003); doi: 10.1117/12.2207497

SPIE Event: Eighth International Topical Meeting on Education and Training in Optics and Photonics, 2003, Tucson, Arizona, United States 


\title{
The optical mouse as an inexpensive measurement device
}

\author{
T.W. Ng \\ Faculty of Engineering EA-07-32, National University of Singapore, 9 Engineering Drive 1, Singapore 117576 \\ engngtw@nus.edu.sg
}

\begin{abstract}
The optical mouse is an inexpensive and easily available device that interfaces directly to a computer. Here, the viability of this device in optical metrology is demonstrated. Application in a wide range of student experiments is anticipated.

(C)2000 Optical Society of America

OCIS codes: (120.4640) Optical Instruments; (120.3940) Metrology
\end{abstract}

\section{Introduction}

Sensing and measurement are the cornerstones of experimentation [1] in science and engineering education. The sensing and measurement of translation is a fundamental endeavor in this respect. However, experiments based on translation using optical sensing are generally expensive to assemble. This has unfortunately resulted in a relative dearth of such experiments. The availability of a cheaper device offers interesting vistas.

Until recently, the operation of computer mice had been based almost exclusively on the rolling ball principle. Popularly called the mechanical mouse, it houses a rubberized ball that rolls according to the planar movement imposed on the mouse. Two rollers located within the mouse are in constant contact with the rubberized ball. One of the rollers detects for motion in the x-direction whereas the other detects for motion in the y-direction. Quite naturally, the mechanical mouse suffers from the problems of wear and dirt accumulation over time. For this reason, it is common to find them incapable of registering movement after several months of heavy usage. In 1999, Agilent Technologies unveiled the first optical mouse that was immune to the problems of wear and dirt accumulation. With resolutions currently reaching $0.03175 \mathrm{~mm}$, optical mice are gradually replacing their mechanical predecessors as the pointing device of choice in computers. Due to the economics of large volume production, the cost of an optical mouse is extremely low. Currently, it is possible to acquire a reasonably good quality unit for as low as US\$20.

Here, the viability of the optical mouse in measurement applications is demonstrated. These applications range from static, quasi-static to dynamic forms.

\section{Incorporation of the optical mouse in translation-based experiments}

Two translation-based experiments were devised to illustrate the possibility of incorporation of the optical mouse as sensing device.

\subsection{Comparison of translation with an opto-mechanical stage}

In this experiment, a xyz translation stage (Newport M-460A-XYZ model) is used. The resolution of the translator along each axis is 10 micrometers. The test surface is attached to the vertical faceplate of the translator (Fig. 1). In the experiment, three types of surfaces were applied for testing: a diffusely white painted plate, a plexiglass sheet, and a square mirror. Each was meant to represent an opaque, transparent, and reflective object respectively. An optical mouse with a resolution of $0.0635 \mathrm{~mm}$ was employed for testing. On the computer, any imaging software (e.g. Windows Paint) may be used to determine the mouse's position. In the experiment, the value of $z$ (Fig. 1) represented the position that the optical mouse was placed from the object's surface. For each setting of $z$, displacements in the $\mathrm{x}$ and $\mathrm{y}$ axis, as registered in the computer due to the optical mouse's movement, were compared with actuations from 0 to $1 \mathrm{~mm}$ at intervals of $0.05 \mathrm{~mm}$ on the xyz translation stage. The same procedure was repeated for values of $\mathrm{z}$ from 0 to $1.5 \mathrm{~mm}$ at increments of $0.25 \mathrm{~mm}$. 


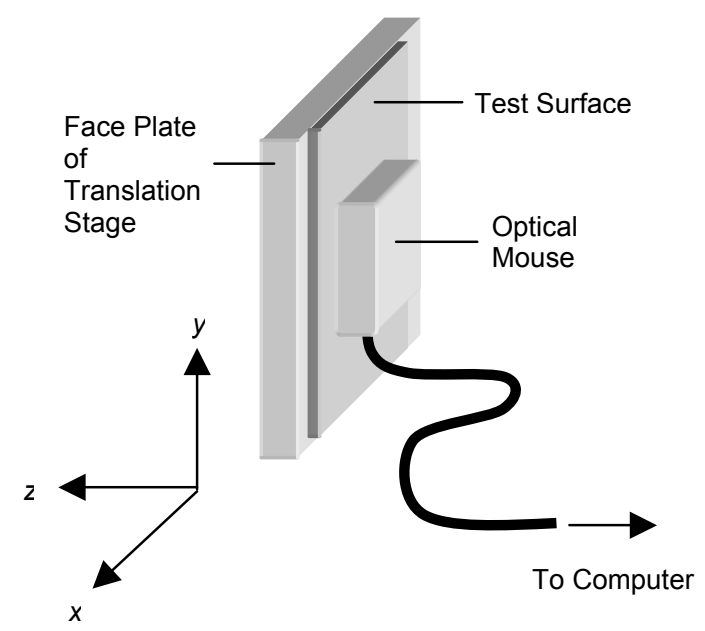

Fig.1. Schematic description of the experiment to compare translation with an opto-mechanical stage

\subsection{Measuring the viscoelastic response of polyethylene}

A very simple demonstration of viscoelasticity can be carried out by attaching a weight to polymer film and watching it extend over time. For quantifiable data, a rule may be stationed beside the polymer film to measure the extension. Such an approach will likely give very coarse measurements that will make it difficult to ascertain important parameters such as the relaxation time. Improved measurements may be obtained via an ultrasonic motion sensor [2] attached to a universal laboratory interface [3]. Such a sensor and interface can be expensive.

In this experiment, the polyethylene film is properly fixed to the top section of a loading fixture (Fig. 2). The lower section of the loading fixture is moveable and comprises an opaque guide. An optical mouse is arranged beside the loading fixture to sense movement of the reference surface. The reference surface should be able to move freely. This can be confirmed by pulling the opaque guide up and down slowly. The gap between opaque guide and optical mouse should also be kept within 1mm; otherwise the optical mouse will fail to register any movement. Once these precautions are adhered to, a weight can be hung at the bottom of the loading fixture. The extension of the polyethylene film is recorded at fixed time intervals (typically 10 seconds) for a certain period of time (typically 5 minutes) using the stopwatch. On the computer, any simple software may be used to determine the mouse's position.

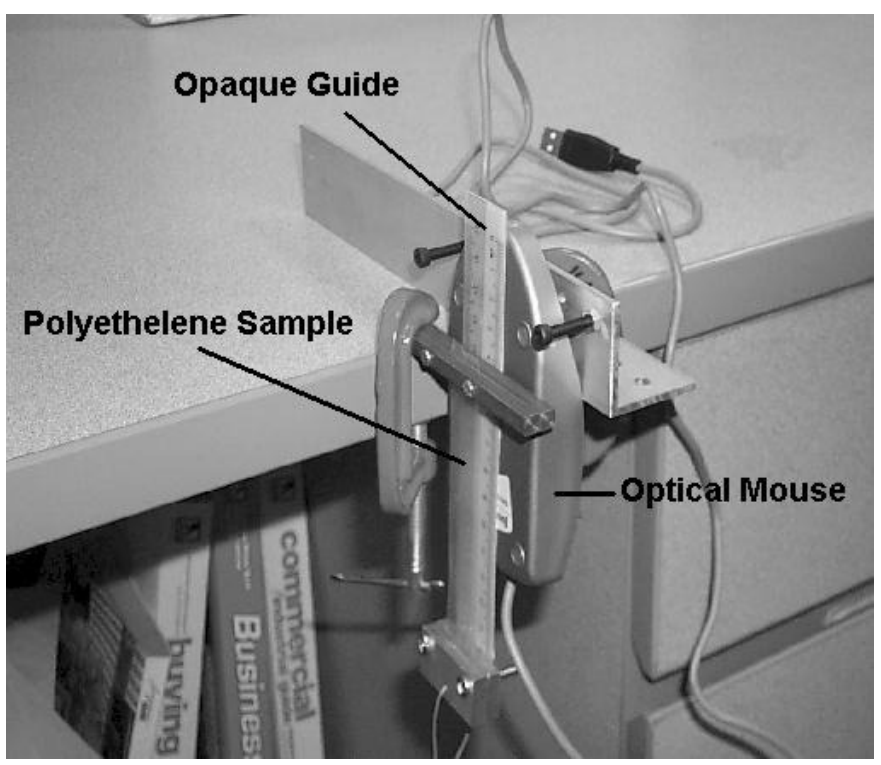

Fig.2. Experiment to determine the viscoleastic response of polyethylene 


\section{Results and Discussion}

In the experiment to compare translation with an opto-mechanical stage, the optical mouse was found to only able to register displacements when the diffuse white painted plate was used as the test object. This result simply confirms that transparent and reflective objects are unsuited for displacement measurement using optical mice.

Fig. 3 shows the graphs of $\mathrm{x}$ and $\mathrm{y}$ direction displacements recorded using the optical mouse and plotted against displacements introduced by the xyz translator for values of $\mathrm{z}=0$ and $\mathrm{z}=0.25 \mathrm{~mm}$. From visual inspection, it can be seen that a positive trend exists between the displacements detected on the optical mouse in relation to the translations introduced. No measurements could be obtained when $\mathrm{z}$ exceeded $1.25 \mathrm{~mm}$. This is likely to be caused by complete defocusing. Together with the observed linear trend, this result implies that the optical mouse is generally suited for two-dimensional displacement measurement provided that $\mathrm{z}$ does not exceed $1.25 \mathrm{~mm}$.

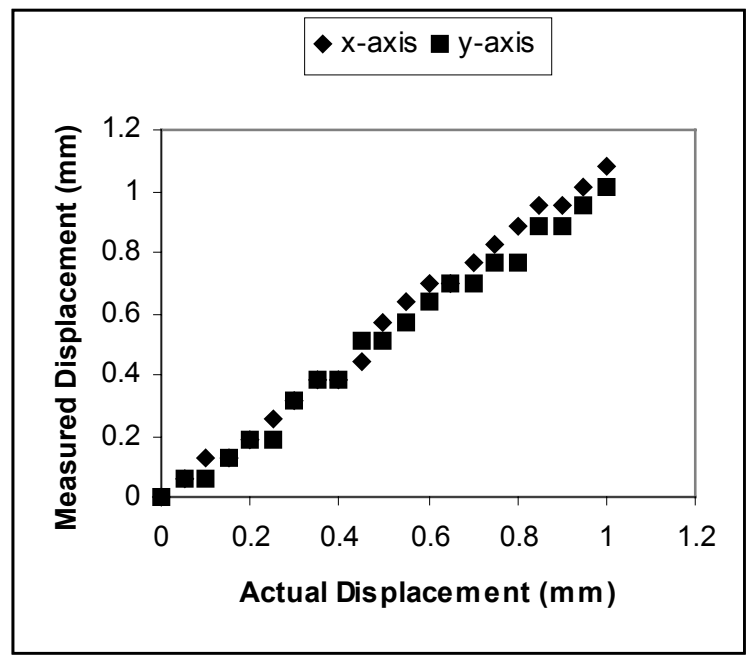

(a)

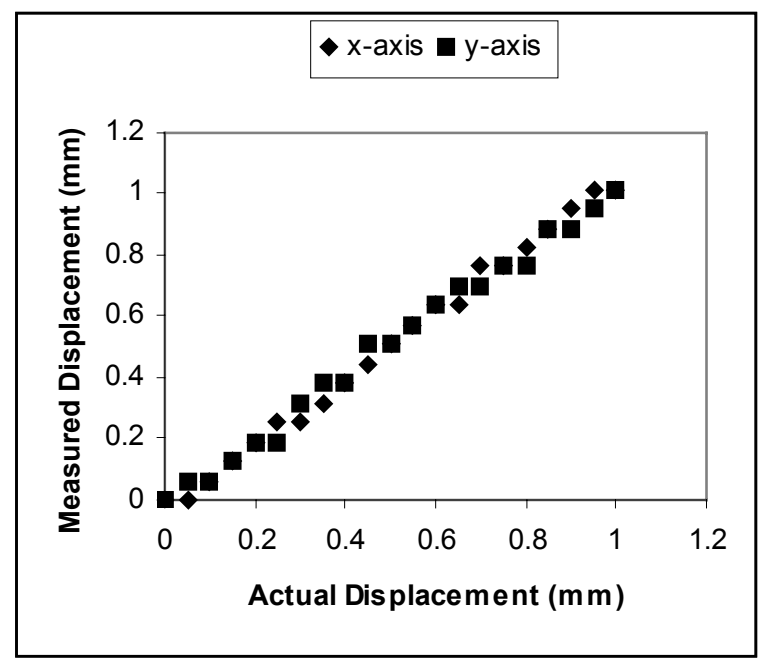

(b)

Fig. 3. Plot of $\mathrm{x}$ and $\mathrm{y}$ direction displacements recorded using the optical mouse against displacements introduced using the translator for (a) $z=0$ and (b) $z=0.25 \mathrm{~mm}$.

The elongation behaviour of low-density polyethylene film in relation to time is given in Fig. 4 . The three films had the same dimension but were obtained from different manufacturers. The trends of the curves follow that reported in literature [4]. This demonstrates the ability of the optical mouse to serve as a displacement sensor in actual engineering applications. Whilst the films displayed clear viscoelastic responses, their displacement amplitudes were clearly different. Such differences can be used to engage in discussions of contributing factors such as processing. The contribution of processing factors is well studied in low-density polyethylene processing [5-6].

It should be noted that the manner in which the experiment was set up prevented movement in the z-direction whilst the film was loaded. Hence, the $\mathrm{z}$ distance limitation mentioned earlier was not operational in this experiment. 


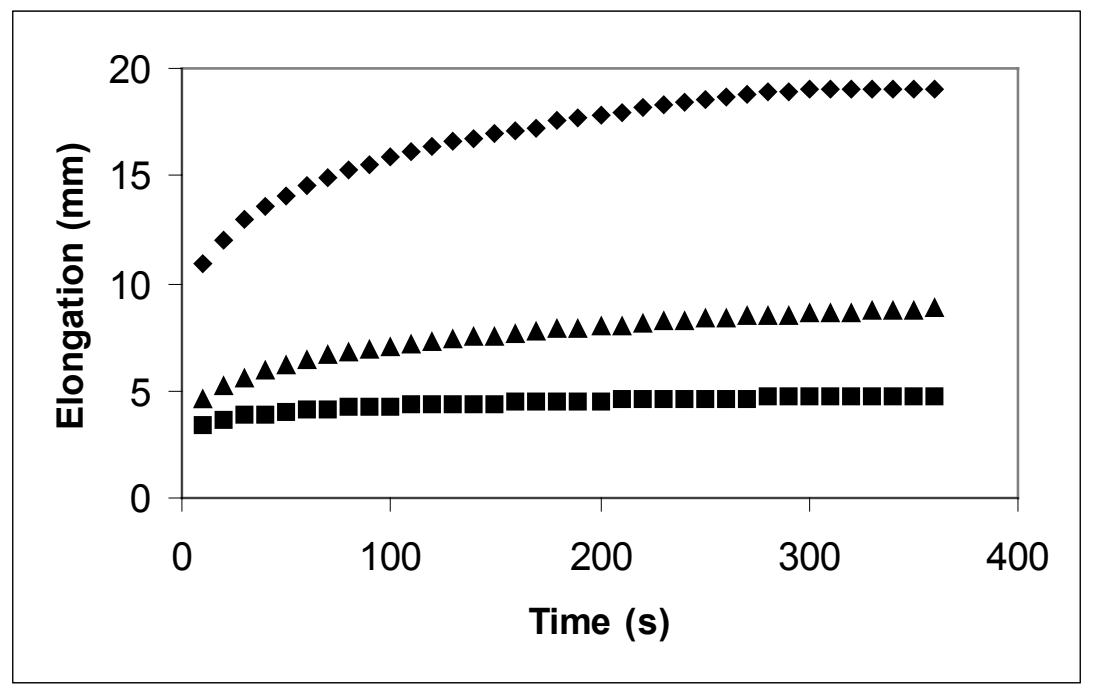

Fig. 4. Plot of elongation against time of the experiment to illustrate the viscoelastic response of polyethylene

\section{Conclusions}

The optical mouse was applied in two experiments; one to compare translation with an opto-mechanical translator and another to sense the viscoelastic response of polyethylene. Its efficacy was proven in both cases. This portends the possibility of creating a wide range of low-cost but engaging experiments for students.

\section{References}

[1] R.S. Fagliola and D.E. Beasley, Theory and design for mechanical measurements (John Wiley, Toronto, 1995).

[2] P.M. Viela, and D. Thompson, "Viscoelasticity: why plastic bags give way when you are halfway home”, European Journal of Physics $\mathbf{1 9 9 9 ,}$ $20,15-20$.

[3] Pasco Scientific http://www.pasco.com.

[4] J. Gittus, Creep, Viscoelasticity and Creep Fracture in Solids (Applied Science, London, 1975).

[5] R.M. Patel, T.I. Butler, K.L. Walton and G.W. Knight, "Investigation of processing structure properties relationships in polyethylene blown films", Polymer Engineering and Science 1994, 34, 1506-1514.

[6] R.K. Krishnaswamy, and M.J. Lamborn, "Tensile properties of linear low density polyethylene blown films", Polymer Engineering and Science 2000, 40, 2385-2396. 